\title{
Network Analysis: Applications for the Developing Brain
}

\section{Citation}

Chu-Shore, C. J., M. A. Kramer, M. T. Bianchi, V. S. Caviness, and S. S. Cash. 2011. “Network Analysis: Applications for the Developing Brain." Journal of Child Neurology 26 (4) (February 7): 488-500. doi:10.1177/0883073810385345.

\section{Published Version}

doi:10.1177/0883073810385345

\section{Permanent link}

http://nrs.harvard.edu/urn-3:HUL.InstRepos:29663461

\section{Terms of Use}

This article was downloaded from Harvard University's DASH repository, and is made available under the terms and conditions applicable to Other Posted Material, as set forth at http:// nrs.harvard.edu/urn-3:HUL.InstRepos:dash.current.terms-of-use\#LAA

\section{Share Your Story}

The Harvard community has made this article openly available.

Please share how this access benefits you. Submit a story.

\section{Accessibility}




\title{
Network Analysis: Applications for the Developing Brain
}

\author{
Catherine J. Chu-Shore, MD, MA ${ }^{1}$, Mark A. Kramer, $\mathrm{PhD}^{2}$, Matt T. Bianchi, MD, PhD ${ }^{1}$, Verne \\ S. Caviness, MD, $\mathrm{PhD}^{1}$, and Sydney S. Cash, MD, PhD ${ }^{1}$ \\ ${ }^{1}$ Department of Neurology, Massachusetts General Hospital, Harvard Medical School, Boston, \\ MA, USA \\ ${ }^{2}$ Department of Mathematics and Statistics, Boston University, Boston, MA, USA
}

\begin{abstract}
Development of the human brain follows a complex trajectory of age-specific anatomical and physiological changes. The application of network analysis provides an illuminating perspective on the dynamic interregional and global properties of this intricate and complex system. Here, we provide a critical synopsis of methods of network analysis with a focus on developing brain networks. After discussing basic concepts and approaches to network analysis, we explore the primary events of anatomical cortical development from gestation through adolescence. Upon this framework, we describe early work revealing the evolution of age-specific functional brain networks in normal neurodevelopment. Finally, we review how these relationships can be altered in disease and perhaps even rectified with treatment. While this method of description and inquiry remains in early form, there is already substantial evidence that the application of network models and analysis to understanding normal and abnormal human neural development holds tremendous promise for future discovery.
\end{abstract}

\section{Keywords}

development; connectivity; network; neocortex

\begin{abstract}
The developing human brain is a dynamic, complex system. Over the course of development, the human brain evolves from a few immature connections to an estimated 20 billion neocortical neurons each with an average 7000 synaptic connections and linked by over $150 \mathrm{~km}$ of myelinated axonal projections. ${ }^{1}$ Historically, rigorous investigation laid the foundation for clinical neurology and systems neuroscience by evaluating restricted or focal components of a system. These reductionist methods are by their nature insensitive to the unique and emergent properties that are evident when evaluating the system as an integrated whole. Over the last decade, many new techniques for exploring complex and dynamic systems have been applied toward understanding the nervous system. Drawing from graph theory, these techniques use information regarding network components and their connections to characterize the overall structure of a system. Analysis of these networks
\end{abstract}

\footnotetext{
(C) The Author(s) 2011

Corresponding Author: Catherine J. Chu-Shore, MD, MA, 175 Cambridge Street, Suite 340, Boston, MA 02114, cchushore@partners.org.

Reprints: http://www.sagepub.com/journalsReprints.nav

Author Contributions

All of the authors were involved in conception of the article, revisions, and in approval of the final version. CJC-S wrote the first draft of the manuscript. MAK wrote the first draft of the section on Analysis of Structural and Functional Networks.

Declaration of Conflicting Interests

The authors declared no potential conflicts of interest with respect to the authorship and/or publication of this article.
} 
offers a rich and tractable means of describing and categorizing the architecture of complex networks, including the developing human brain. The wide utility of these approaches facilitates applications in neuroscience that include characterizing anatomical relationships between brain regions as well as temporally correlated activity patterns in different brain regions. Network methods are especially valuable for child neurologists, given the profound changes that occur throughout neurodevelopment in both normal and pathological circumstances.

In this review, we will highlight recent work analyzing neural networks of the normal developing brain as well as potential clinical applications. We will focus on 4 related topics: basic attributes of network analysis with a particular focus on functional brain networks, a brief overview of the primary histogenetic events and sequences of anatomical cortical development, an examination of what is known for the development of functional cortical networks, and the clinical implications of these findings as well as future directions.

\section{Analysis of Structural and Functional Networks}

Many examples of networks exist in the world. These include structural networks (eg, the network of roads that connect cities and towns), social networks (eg, the networks of film actors that collaborate in movies), and biological networks (eg, a network of neurons connected with synapses). In each instance, we can represent the network with 2 fundamental components: nodes (the cities, actors, or neurons) and edges (the roads, films, or synapses) that connect any 2 nodes. In what follows, we will focus on binary, undirected networks. In these simplified networks, an edge either exists between 2 nodes or does not, and the presence of an edge provides no directional (ie, causal) information. In neuroscience, networks are typically divided into 2 categories: structural networks and functional networks. ${ }^{2}$ In structural networks, the edges represent physical connections between nodes. At the microscopic spatial scale, these include synaptic or gap junctional connections between individual neurons. ${ }^{3,4}$ At the macroscopic spatial scale, white matter tracts are used to infer synaptic connections between brain regions and construct structural networks. ${ }^{5-7}$

A functional network is defined by analysis of dynamic physiological activities, rather than by anatomy per se. Any time-varying physiological signal, such as electrical activity, magnetic activity, or blood oxygenation, can be the basis for inferring a functional network of brain activity. Generating the network requires recording such signals from multiple spatial locations and a metric to analyze coupling between these signals. The most commonly available clinical measure of brain activity is the routine electroencephalogram (EEG). To identify a functional network from the EEG, a coupling measure is applied to pairs of voltage signals recorded at separate EEG electrodes. Strong coupling between the activities recorded at 2 EEG electrodes defines an edge in the network, and the EEG sensors define the network nodes.

Figure 1 illustrates this process in a simplified case of EEG data recorded from 2 scalp electrodes. To determine whether the 2 recording sites are linked (or connected) in this 2node network, we must determine the extent to which the EEG data recorded at the 2 electrodes match. There is no single method to optimally characterize signal coupling and many choices exist. ${ }^{8}$ Here, we demonstrate the process with a simple measure called the cross correlation. Cross correlation compares the temporal patterns contained in the 2 signals over time to see how well the voltage fluctuations align. One way to accomplish this is to compare the signals directly (the trace of signal 1 versus signal 2 at each time point) as well as indirectly: we can shift the traces in time with respect to one another to determine if the signals match more strongly at a particular lead or lag. If at some time shift the 2 signals 
match, then the cross correlation between the 2 signals is strong and we represent this graphically in a network by drawing an edge between the 2 electrodes (Figure 1A). On the other hand, if the 2 signals never match, no matter our choice of time shift, then the cross correlation between the 2 signals is weak and we leave the 2 electrodes disconnected in the network representation (Figure 1B). In this simple illustration, we have described a 2-node network that either contains 1 edge (Figure 1A) or does not (Figure 1B). The 2 nodes represent the 2 EEG electrodes, and the edge reflects correlated activity (ie, coupling) between the voltage fluctuations recorded from the 2 electrodes. This approach can be extended to build larger networks by applying coupling measures to the voltage signals recorded from all electrode pairs, for example, in routine 10-20 configuration EEGs (Figure 1C). These results can then be used to create a functional connectivity network for the entire EEG montage (Figure 1D).

Having constructed a functional network, we now seek to analyze its structure. A useful, first analysis is simple visual inspection of the network (eg, visual inspection of the network in Figure 1D). Even in this 10-20 configuration, the network structure becomes difficult to visualize. In fact, as the number of nodes in a network increases, so does the number of possible edges, and visual inspection becomes less useful. For a network of $\mathrm{N}$ nodes, the maximum possible number of edges is $\mathrm{N}(\mathrm{N}-1) / 2$. When $\mathrm{N}$ is large (eg, in a high density EEG recording with 128 electrodes or a multielectrode array with 100 contacts) the networks typically become much too complicated for visual inspection (Figure 2A, B). To go beyond visual inspection and characterize the structure of these large networks, many measures exist. ${ }^{9-11}$ Here, we outline 3 of these measures in common use: the degree, path length, and clustering coefficient.

The degree (d) is simply the number of edges that touch a node. In Figure 3, we show a 5node network, and list the degree of each node (in this case, a value of either 2 or 4). To summarize the degree values of the entire network, we compute the average degree of all nodes, and find in this case 2.4. We note that this average value lies between the degree values we observe for each node ( 2 or 4 ), as expected. For much larger networks, the degree distribution is a useful measure that illustrates the probability of observing a node of degree $\mathrm{d}$ (Figure 2C). In many real world networks, including the film actor network and neural networks, ${ }^{12}$ the degree distribution exhibits a power law: the probability of observing a node of degree d decreases as 1 over the degree to some power (Figure 2D). ${ }^{13}$ In these networks, high degree nodes (which appear less frequently than the low degree nodes) can serve important functional roles in the network (ie, can act as hubs) although this is not always the case. ${ }^{14}$ Degree distributions with power law behavior are also known as scale-free because the degree distribution looks the same (just scaled by constant value) if we multiply the value of $d$ by a constant.

The path length is the minimum number of edges traversed to go from any given node to another in the network. We assume that each node is reachable from any other node, but if this is not the case, care must be taken to adjust for unreachable nodes. In the example 5node network, the path length from node i to any other node is 1 ; node i can reach any other node by traversing 1 edge. Nodes ii-v can reach any other node in 1 or 2 steps. We note that many different paths exist between nodes. For example, we can travel directly from node ii to node iii, or we can pass through node $\mathrm{i}$ on the way to node iii. When computing the path length, by convention we always choose the shortest path between nodes. The average path length is calculated from the path length between each node and all other nodes in the network; for the 5-node network, the average path length is 1.4, a value between 1 and 2, as expected. 
The final measure we consider here is the clustering coefficient. The clustering coefficient of a node is the number of connections that exist between the nearest neighbors of a node, expressed as a proportion of the maximum number of possible connections between the nearest neighbors of the node. In this context, nearest neighbor is not a spatial measure but a measure of which nodes are connected. This definition is perhaps best illustrated through the example network in Figure 3. In the 5-node network, choose node ii and notice that this node has 2 neighbors (ie, 2 nodes directly connected to node ii by a single edge, nodes $i$ and iii in Figure 3B). We now examine whether an edge exists between the 2 neighbor nodes. In this example, it does (see Figure 3A) so we complete a triangle or cluster in the network. In social networks, clustering is typically high; the friends (nearest neighbors) of an individual (the chosen node) also tend to be friends (ie, edges connect the nearest neighbors of the chosen node). For node $\mathrm{i}$ in the 5 -node network, the clustering coefficient is $1 / 3$. This result indicates that of all the possible completed triangles between the nearest neighbors of node $i$, only one-third exist. To complete all of the triangles between the nearest neighbors of node $i$ would require additional connections between nodes ii and iv, nodes ii and v, nodes iii and iv, and nodes iii and v. For all other nodes, the clustering coefficient equals 1 . All possible triangles between the nearest neighbors of these other nodes do exist. Often the average clustering coefficient for all nodes in a network is computed; for the 5-node network, the average clustering coefficient is $13 / 15$.

With even these 3 simple measures in hand, we can characterize an arbitrarily large and complicated network easily and can compare networks efficiently. These, and other measures, therefore allow us to reduce the potentially overwhelming complexity of a developing brain network into a few comparable and informative measures. The next sections outline the maturation of anatomical and functional brain networks at each stage of development. We examine the application of network analysis to track the complex, but orderly, network topologies over neurodevelopment.

\section{Development of Neocortical Anatomical Connectivity}

The human cortex develops its familiar laminated architecture primarily in utero. ${ }^{15,16}$ During the first 2 to 6 months of gestation, ${ }^{17}$ projection neuron precursors undergo massive proliferation in the ventricular zone, ${ }^{18-23}$ amplified by secondary divisions in the subventricular zone. ${ }^{24,25}$ Simultaneously, neuronal precursors migrate radially to form the early cortical plate. Directed along a glial scaffolding and guided by regulatory gene expression, these cortical neurons distribute primarily in an inside-to-outside pattern based on time of origin. ${ }^{21,22}$ Inhibitory interneurons, by contrast, arise in the ganglionic eminences and reach their cortical positions by means of tangential migrations. ${ }^{18,19}$ The distinctive laminar and columnar arrays of the 6-layered mature neocortex are evident by 8 months gestation. ${ }^{22,26,27}$

Cortical development continues to evolve after birth, and extensive work has described changes in white and gray matter from infancy through adolescence. Longitudinal MRI studies demonstrated that cortical gray matter volume increases through childhood to puberty, after which there is a net loss of volume. ${ }^{28}$ This predictable sequence is observed in an inverse-hierarchical manner, such that changes in primary motor and sensory regions precede the association cortices and prefrontal cortices that mediate executive functions ${ }^{29,30}$ (see Figure 4).

The mechanisms underlying the inverted U-shaped temporal pattern of cortical volume are diverse $^{31}$ (see Figure 5). The initial proliferation during fetal development is later sculpted by selective cell death. ${ }^{17,32-35}$ Heightened synaptogenesis beginning in the third trimester continues through 2 years of age but then is followed by a prolonged period of dendritic 
pruning which extends through childhood and adolescence. The final synaptic density is approximately $60 \%$ of the maximum values. ${ }^{33}$ Similar to patterns of cortical volume change, synaptic density reaches a maximum in primary sensory cortical regions before association cortices. For example, in the primary auditory cortex maximal synaptic density is observed around 3 months of age, approximately 1 year earlier than maximal synaptic density is reached in the prefrontal cortex. ${ }^{33}$ In addition, deeper cortical layers (4, 5, and 6) develop more rapidly than superficial layers ( 2 and 3 ) ${ }^{33}$ The cortical volume loss evident on MRI correlates in time with increased synaptic pruning, as observed in pathology specimens of neurologically normal subjects.

In contrast to the expansion and pruning patterns of cortical gray matter described above, ${ }^{36,37}$ cerebral white matter volume grows steadily over time, largely attributed to intra-cortical fiber myelination. Myelination improves impulse conduction to such an extent that signal velocity is primarily determined by segments of unmyelinated intracortical conduction length. ${ }^{38}$ Myelination begins during the second trimester of fetal development and increases over the first 2 years of life. ${ }^{39}$ Myelination continues throughout the first 3 decades of life, but at a much slower pace. ${ }^{40}$ The temporal sequence of white matter myelination is similar to that of cortical development, with fibers mediating primary sensory then motor systems myelinated first (in infancy), followed by association and then prefrontal cortices (in childhood). ${ }^{41,42}$

White matter tract visualization can be accomplished in vivo with an MRI-based technique, diffusion tensor imaging. A full review of diffusion tensor imaging and related techniques is beyond the scope of this article but can be found in several excellent reviews. ${ }^{43,44}$ Briefly, the preferred direction of water diffusion is used to define white matter tracts by a metric called fractional anisotropy, even in unmyelinated fibers in immature brains. ${ }^{45}$ Recent work has demonstrated that diffusion tensor imaging can even distinguish glial fiber density between fetal cortical layers effectively. ${ }^{46}$

Longitudinal studies of normal development have shown that prominent white matter tracts can be visualized at birth, although fractional anisotropy values are lower in the immature, incompletely myelinated white matter. ${ }^{47,48}$ As myelination occurs, water diffusion becomes more directional with an accompanying dramatic increase in fractional anisotropy values over the first 2 years. Adult values are generally obtained by 4 years of age. Although the corpus callosum and anterior commissure are detected by diffusion tensor imaging in newborns, association fibers (superior longitudinal fasciculus, inferior longitudinal fasciculus, and inferior fronto-occipital fasciculus) are not observed until 3 to 12 months of age $^{47}$ (see Figure 6). Localized age- and region-specific maturation of discrete white matter tracts continues through adolescence. ${ }^{49}$

Network topology has been evaluated using diffusion tensor imaging-based connectivity graphs. Evaluation of cortico-cortical anatomical connections reveals high clustering and short characteristic path lengths, typical of "small-world" networks. ${ }^{50,51}$ In such networks, most nodes can be connected through only a small number of steps, reflecting a high efficiency of information transfer, computational power, and synchronizability across the network. ${ }^{2,12}$ Small-world topology of brain anatomical networks is evident at 1 year of age and increased efficiency and modular organization develops over the first 2 years. ${ }^{52}$ Over adulthood, network efficiency decreases, possibly due to age-dependent white matter degeneration. ${ }^{53}$

Anatomical connectivity patterns are disrupted in a variety of acquired, genetic, and idiopathic neurodevelopmental disorders. ${ }^{54-57}$ Much future work, characterizing the 
relationship between network topology and clinical symptoms in pediatric neurodevelopmental disorders remains to be done.

The developing anatomical networks reviewed here provide the physical substrate for functional connections. Below we describe observed functional network topologies over early development in both normal and pathological circumstances.

\section{Development of Neocortical Functional Connectivity}

Physiological interactions among brain regions are influenced and constrained by the underlying anatomical frameworks that arise from the developmental processes discussed above. ${ }^{58}$ Similarly, spontaneous and experience-dependent neural activity guides all aspects of architectural development, from neurogenesis, neuronal differentiation, and migration to synaptogenesis and dendritic pruning. ${ }^{59-66}$ Thus, anatomy and physiology interdependently direct the development, sculpting, and stabilization of cortical functional networks. With the advent of new measures for determining functional connectivity and analysis of networks, these physiological connectivity patterns can now be observed over development.

As described above, networks can be constructed from correlated activity between EEG voltage traces. These networks are taken to reflect both (relatively) static, anatomical connectivity between the cortical neuronal populations being measured and dynamic connectivity between active neural populations. Functional connectivity networks derived from EEG therefore serve to capture anatomically mediated state-dependent, dynamic and shifting physiological networks. One recent study evaluated synchronization patterns between all possible electrode pairs in routine scalp EEG in developmentally normal children ages 5 and 7 years while at rest. ${ }^{67}$ The older children demonstrated an overall decrease in functional connectivity and increases in both average clustering and path length, which the authors speculated could be related to synaptic pruning. High-density (128channel) EEG has been used to investigate differences in coherence between EEG channels of school-aged children compared with young adults in the alpha frequency $(8-12 \mathrm{~Hz})$ range. ${ }^{68}$ Local coherence between nearby pairs of electrodes was similar between the groups. However, young adults exhibited increased coherence between longer range pairs of electrodes, in particular between anterior and posterior electrodes. Similar findings have been reported by other groups, ${ }^{69,70}$ possibly reflecting increased myelination of longdistance fasciculi or increased small-world properties in the older maturing brain.

Another technique for probing functional connectivity patterns involves use of sensory stimulation to perturb or trigger physiological signals (or evoked responses) and measure phase synchronization of the signals by EEG. Delta brushes, characterized by asynchronous delta activity $(0.3-1.5 \mathrm{~Hz})$ with overriding faster $(8-22 \mathrm{~Hz})$ frequencies are normal features of the EEG recording in premature infants. ${ }^{71}$ Spontaneous movements and sensory stimulation have been shown to evoke somatotopically organized delta brushes in the contralateral sensorimotor cortical region of premature infants, possibly reflecting early organization of sensory neural circuits. ${ }^{72}$ By 8 months of age, bursts of gamma frequency activity $(\sim 40 \mathrm{~Hz})$ can be evoked in the left frontal region in response to complex visual stimuli. ${ }^{73}$ Gamma oscillations can be related to the process of "binding," or conceptual association of sensory inputs, and in this case can be involved in the development of complex visual processing. Age-specific changes in visually to evoked gamma oscillation patterns have been demonstrated from childhood through young adulthood. ${ }^{60,74}$

Other frequency bands also demonstrate maturation in visual stimulus-evoked synchrony, as one group demonstrated with high density EEG over the age range of 6 to 21 years. ${ }^{73}$ For example, a period of relative desynchronization in the beta band $(12-16 \mathrm{~Hz})$ correlated with task performance (stimulus detection rate and reaction time to stimulus onset), with 
adolescents showing both decreased synchronization and lower performance compared with other age groups. Furthermore, younger age groups demonstrated widespread synchrony whereas the older group showed more focal synchronization patterns. Such age-specific dynamics suggest that the normal maturation of functional cortical connections likely involves complex system dynamics rather than a simple incremental process. Although these groups did not map synchronization patterns into a network space, their findings are in essence an unstructured map with altered degrees of connectivity. Future work involving formal application of graph theoretic approaches will allow for further characterization of these functional relationships.

One area of intense research involves measuring brain activity under task-free or "resting" conditions. This so-called "default" network shows activity that is higher at rest than during tasks requiring attention, ${ }^{75-78}$ although there is ongoing discussion of the meaning and definition of this network. ${ }^{79}$ Low frequency $(<0.1 \mathrm{~Hz})$ fluctuations in blood oxygenation level-dependent signals can be treated similarly to EEG voltage fluctuations, with correlations measured between voxels or brain regions of interest. Resting activity is obtained while a subject is awake, usually with eyes closed and without any particular task or instruction, such that the blood oxygenation level-dependent fluctuations are thought to indirectly reflect spontaneous neural activity. Brain regions of interests are considered as nodes in a network in the same sense that EEG channels can be, and the connections between the nodes are determined by the strength of the coupling between blood oxygenation level-dependent signals.

Functional connectivity in the default network has been measured across development using functional connectivity MRI in quiet or resting states. Even in preterm infants, resting state functional connectivity MRI identified 5 networks, in this case observed during sleep. ${ }^{80}$ This activity can represent a precursor to the default network seen in adults (which also persists into sleep, interestingly ${ }^{81}$ ). In preterm infants, coherence was prominent between homotopic cortical regions likely mediated by the relatively mature underlying transcallosal white matter tracts in this age group. A separate study similarly identified a putative precursor to the default network in term infants. ${ }^{82}$ Of interest, there was no indication of default network activity in preterm infants scanned at term-equivalent. The gradual maturation of the default functional network is further suggested by the finding of an intermediate, sparsely connected network profile evident in school age children, distinct from the pattern seen in infancy and the mature form of adulthood ${ }^{83}$ (Figure 7). The apparent incremental evolution of the default network over development and the relative persistence of this network through wakefulness and sleep suggest that it can reflect the robustness of underlying structural connectivity patterns as opposed to less stable, state-dependent, shifting physiological frameworks, which can be more apparent in modalities with higher temporal resolution.

Near-infrared spectroscopy is a related indirect metric of neuronal activity, which measures cerebral blood oxygenation by means of hemoglobin saturation content. Like blood oxygenation level-dependent fluctuations of functional MRI, fluctuations in near-infrared spectroscopy signals can also be used to infer correlated activity between brain regions. Using this method, functional connectivity networks have been defined by correlated nearinfrared spectroscopy fluctuations during sleep in infants 0 to 6 months of age. ${ }^{84}$ Connectivity between homotopic temporal regions was evident by 3 months of age and persisted. In addition, high coherence between parietal and temporal regions on the left was seen at 6 months of age, which the authors speculated can be related to the development of early language networks.

Another example of immature versions of adult connectivity patterns was evident in a resting state functional connectivity MRI study of school-aged children. Decreased 
segregation between, as well as decreased connectivity within, 2 previously identified adult attention networks (fronto-parietal and cingulo-opercular) ${ }^{85}$ was found in this age group. ${ }^{86}$ Older children, in the adolescent range (10 to 15 years) showed an intermediate network structure. Older children also had more long-range connections and less short-range connections. The authors suggest that developmental remodeling of these 2 networks contributes to the acquisition of task controlling behaviors.

Graph theory analysis methods applied to functional networks are also proving useful for quantifying developmental changes in activity patterns defined by resting state functional connectivity MRI ${ }^{87}$ Small-world properties (networks with high clustering coefficient and low path length, as described above) were observed in children aged 7 to 9 years as well as young adults age 19 to 22 years. Also, a measure of global efficiency, the harmonic mean of the minimum path length between each pair of nodes, was similar between these age groups. Differences were however notable in cortical-subcortical connectivity, which was greater in children. In addition, young adults exhibited increased network hierarchy (characterized by the presence of small densely connected clusters that combine to form large lessinterconnected clusters) and stronger cortico-cortical connections. These attributes are consistent with the development of advanced network specialization with age. The finding of increased distant and decreased local connections in adolescents could reflect the combined influence of improved myelination and relative weakening of local, nonspecific connections as networks become more specialized and distributed.

Not all studies of functional connectivity support or parallel gross anatomical development. For example, beta frequency activity measured in a large cohort ranging in age from infants to adolescents reported an initial increase in connectivity between all electrode pairs followed by a sharp decline by age $\sim 3$ years, and then a steady rise in short-range connections (6-12 cm in this study) subsequently.$^{88}$ The suggested decrease in long-distance connections over development appears to contrast with myelination patterns and EEG ${ }^{68-70}$ and resting state functional connectivity MRI work above. ${ }^{86,87,89}$ This discrepancy highlights the challenges in comparing functional networks defined by different measures of neuronal activity such as EEG versus functional MRI, which use distinct frequency preferences (lower in functional MRI) and distinct anatomical resolutions (higher in functional MRI), as well as distinct analysis strategies (only intrahemispheric coherence versus intra- and interhemispheric coherence, for example). Although further studies, particularly simultaneous EEG and functional MRI recordings, can shed some light on these differences, it is worth noting that, unlike anatomically defined networks, there is no gold standard against which to compare functional connectivity networks.

\section{Clinical Implications}

Although many questions remain regarding how brain networks change over the course of development, the tools and approaches hold tremendous promise for future discovery. Improved characterization of the developmental changes in brain network connectivity, by anatomical and functional measurements, is beginning to provide a framework for understanding how dysregulation at various stages can cause significant neurodevelopmental pathology. Although only a few investigations have been reported thus far, the techniques of network analysis can prove useful for children suffering from neurological disorders, whether through improved phenotypic characterization, natural history prediction, or, ideally, improved treatment strategies.

From the standpoint of phenotypic characterization, one study used functional MRI during an auditory listening task in 8-year-old children with a history of premature birth as well as low intelligence scores. ${ }^{90}$ Increased functional connectivity was observed among language 
processing regions (Wernicke's area, right inferior frontal gyrus, left and right supramarginal gyri, and components of the inferior parietal lobules). It remains unknown whether the abnormal functional connectivity caused the cognitive impairment, resulted from it, or whether both observations are merely epiphenomena.

Despite the limitations of extrapolating mechanism from correlational studies, in many cases, the network abnormalities appear reasonably linked to clinical observations. For example, adolescents with Tourette syndrome exhibit immature functional connectivity patterns in networks thought to be involved in impulse control, such as fronto-parietal and cinguloopercular networks. ${ }^{91}$ Both resting and task-related functional MRI studies in untreated children diagnosed with attention deficit hyperactivity disorder revealed decreased functional connectivity in the cortical-striatal-thalamic circuit, and increased connectivity in the orbitofrontal and superior temporal cortices. ${ }^{92}$ Observed network abnormalities improved or reversed with treatment, ${ }^{93}$ highlighting the potential utility of network analysis for treatment monitoring and/or response prediction.

The clinical suggestion that a common theme in autism spectrum disorders is disrupted sensory integration matches well with the concepts emphasized in functional connectivity approaches. ${ }^{94}$ Consistent with abnormal connectivity, diffusion tensor imaging studies reveal white matter abnormalities in the corpus callosum, superior temporal gyrus, and temporal stem in patients with autism spectrum disorders. ${ }^{95,96}$ Similarly, functional MRI network connectivity studies show overall weaker functional connectivity in the resting state as well as during a social attribution task. ${ }^{97}$ Further studies have identified decreased functional connectivity in specific regions, including the insula ${ }^{98}$ and multiple default mode sub-networks ${ }^{99}$ in patients with autism spectrum disorders. Importantly, the extent or severity of the clinical syndrome (measured across social interactions, behavioral restrictions, and language difficulties) correlated with functional connectivity abnormalities, including the default network. ${ }^{99-101}$ Functional connectivity patterns, however, were independent of treatment status, suggesting a possible role for these biomarkers to guide treatment selection ${ }^{99}$.

At present, only a single study has evaluated functional connectivity networks in children with epilepsy. ${ }^{102}$ Twelve children with continuous spike and waves during slow wave sleep, an epileptic encephalopathy typically characterized by epileptiform discharges during at least $85 \%$ of slow wave sleep and progressive cognitive deficits, were evaluated with simultaneous EEG and functional MRI. Although individual differences were noted, all children were found to have activation of bilateral perisylvian regions and the cingulate gyrus. Since epilepsy is a disorder of aberrant neurophysiology, functional connectivity networks analysis can provide a sensitive clinical tool to track and better understand the pathophysiological impact of altered neurophysiology during critical periods of neurodevelopment.

Although research in this field is still at the earliest stages, these early studies identify network analysis as an important toolkit with potential applicability ranging from diagnostic biomarkers to the prognosis and treatment guidance in pediatric neurodevelopmental disorders.

\section{Conclusions}

The developing human brain follows a complex and tightly regulated trajectory of dramatic anatomical and physiological changes. The majority of studies in both animals and humans have examined focal abnormalities or only specific components of the brain from embryogenesis on. The establishment and continued advancement of network measures 
provide a tractable method to evaluate and compare the dynamic global and interdependent properties present in this complex, plastic system taken as a whole. As new technologies that allow for precise characterization of the evolution of functional and anatomical networks in the immature brain are increasingly available, investigation of developmental brain networks is exploding with rapid advances in both basic science and therapeutic applications. Work in this field has thus far demonstrated that, at each stage in normal neurodevelopment, agespecific skill sets correlate with age-specific distributed brain networks, which progress in a nonlinear, nonincremental, and yet predictable manner. Ultimately, insights from these approaches can offer new opportunities for early detection, prognostication, and guided clinical intervention in a variety of neurodevelopmental disorders. Further work will help us to better understand the short and long-term clinical consequences of focal and global network disruptions in the developing brain, and perhaps reveal new pathways to treatment.

\title{
Acknowledgments
}

\author{
Financial Disclosure/Funding
}

The authors disclosed receipt of the following financial support for the research and/or authorship of this article: Mark A. Kramer holds a Career Award at the Scientific Interface from the Burroughs Well-come Fund. Matt T. Bianchi receives funding from the Department of Neurology, Massachusetts General Hospital, and the Clinical Investigator Training Program: Harvard/MIT Health Sciences and Technology—Beth Israel Deaconess Medical Center, in collaboration with Pfizer, Inc., and Merck \&Co. Sydney S. Cash is supported by NINDS-NS062092.

\section{References}

1. Drachman DA. Do we have brain to spare? Neurology. 2005; 64:2004-2005. [PubMed: 15985565]

2. Bullmore E, Sporns O. Complex brain networks: graph theoretical analysis of structural and functional systems. Nat Rev Neurosci. 2009; 10:186-198. [PubMed: 19190637]

3. White J, Southgate E, Thomson, S. Brenner. The structure of the nervous system of the nematode Caenorhabditis elegans. Philos Trans R Soc Lond B Biol Sci. 1986; 314:1-340.

4. Achacoso, TB.; Yamamoto, WS. AY's Neuroanatomy of C. elegans for Computation. Boca Raton: CRC Press; 1992.

5. Iturria-Medina Y, Canales-Rodriguez EJ, Melie-Garcia L, et al. Characterizing brain anatomical connections using diffusion weighted MRI and graph theory. Neuroimage. 2007; 36:645-660. [PubMed: 17466539]

6. Iturria-Medina Y, Sotero RC, Canales-Rodriguez EJ, Aleman-Gomez Y, Melie-Garcia L. Studying the human brain anatomical network via diffusion-weighted MRI and Graph Theory. Neuroimage. 2008; 40:1064-1076. [PubMed: 18272400]

7. Hagmann P, Cammoun L, Gigandet X, et al. Mapping the structural core of human cerebral cortex. PLoS Biol. 2008; 6:e159. [PubMed: 18597554]

8. Pereda E, Quiroga RQ, Bhattacharya J. Nonlinear multivariate analysis of neurophysiological signals. Prog Neurobiol. 2005; 77:1-37. [PubMed: 16289760]

9. S. Wasserman, KF. Social Network Analysis: Methods and Applications. New York: Cambridge University Press; 1994.

10. Newman M. The structure and function of complex networks. SIAM Rev. 2003; 45:167-256.

11. Kolaczyk, E. Statistical Analysis of Network Data: Methods and Models. New York: Springer; 2009.

12. Watts DJ, Strogatz SH. Collective dynamics of 'small-world' networks. Nature. 1998; 393:440442. [PubMed: 9623998]

13. Albert R, Barabási A. Statistical mechanics of complex networks. Rev Mod Phys. 2002; 74:47-97.

14. Doyle JC, Alderson DL, Li L, et al. The "robust yet fragile" nature of the Internet. Proc Natl Acad Sci U S A. 2005; 102:14497-14502. [PubMed: 16204384]

15. Brodmann, K. Vergleichende Lokalisationslehre der Grosshirnrinde. Leipzig: Barth; 1909. 
16. C von Economo, KG. Vergleichende Lokalisationslehre der Grosshirnrinde. Berlin: SpringerVerlag; 1925.

17. Herschkowitz N. Brain development in the fetus, neonate and infant. Biol Neonate. 1988; 54:1-19. [PubMed: 3061475]

18. Anderson SA, Kaznowski CE, Horn C, Rubenstein JL, McConnell SK. Distinct origins of neocortical projection neurons and interneurons in vivo. Cereb Cortex. 2002; 12:702-709. [PubMed: 12050082]

19. Kriegstein AR. Constructing circuits: neurogenesis and migration in the developing neocortex. Epilepsia. 2005; 46 Suppl 7:15-21. [PubMed: 16201991]

20. Sauer F. Mitosis in the neural tube. The Journal of Comparative Neurology. 1935; 62:377-405.

21. Sidman RL, Miale IL, Feder N. Cell proliferation and migration in the primitive ependymal zone: an autoradiographic study of histogenesis in the nervous system. Exp Neurol. 1959; 1:322-333. [PubMed: 14446424]

22. Rakic, RSaP. Development of the human central nervous system. In: Adams, RD.; Haymaker, W., editors. Histology and Histopathology of the Nervous System. Springfield, IL: Charles C Thomas; 1982. p. 3-145.

23. His, W. Die Entwicklung des Menschlichen Gehirns warhrend der ersten Monate. Leipzig: Hirzel; 1904.

24. Kowalczyk T, Pontious A, Englund C, et al. Intermediate neuronal progenitors (basal progenitors) produce pyramidal-projection neurons for all layers of cerebral cortex. Cereb Cortex. 2009; 19:2439-2450. [PubMed: 19168665]

25. Noctor SC, Martinez-Cerdeno V, Ivic L, Kriegstein AR. Cortical neurons arise in symmetric and asymmetric division zones and migrate through specific phases. Nat Neurosci. 2004; 7:136-144. [PubMed: 14703572]

26. Mountcastle VB. The columnar organization of the neocortex. Brain. 1997; 120(Pt 4):701-722. [PubMed: 9153131]

27. Osheroff H, Hatten ME. Gene expression profiling of preplate neurons destined for the subplate: genes involved in transcription, axon extension, neurotransmitter regulation, steroid hormone signaling, and neuronal survival. Cereb Cortex. 2009; 19 Suppl 1:i126-i134. [PubMed: 19398467]

28. Giedd JN, Blumenthal J, Jeffries NO, et al. Brain development during childhood and adolescence: a longitudinal MRI study. Nat Neurosci. 1999; 2:861-863. [PubMed: 10491603]

29. Gogtay N, Giedd JN, Lusk L, et al. Dynamic mapping of human cortical development during childhood through early adulthood. Proc Natl Acad Sci U S A. 2004; 101:8174-8179. [PubMed: 15148381]

30. Durston S, Hulshoff Pol HE, Casey BJ, Giedd JN, Buitelaar JK, van Engeland H. Anatomical MRI of the developing human brain: what have we learned? J Am Acad Child Adolesc Psychiatry. 2001; 40:1012-1020. [PubMed: 11556624]

31. Casey BJ, Tottenham N, Liston C, Durston S. Imaging the developing brain: what have we learned about cognitive development? Trends Cogn Sci. 2005; 9:104-110. [PubMed: 15737818]

32. Finlay BL, Wikler KC, Sengelaub DR. Regressive events in brain development and scenarios for vertebrate brain evolution. Brain Behav Evol. 1987; 30:102-117. [PubMed: 3304526]

33. Huttenlocher PR, Dabholkar AS. Regional differences in synaptogenesis in human cerebral cortex. J Comp Neurol. 1997; 387:167-178. [PubMed: 9336221]

34. Finlay BL, Slattery M. Local differences in the amount of early cell death in neocortex predict adult local specializations. Science. 1983; 219:1349-1351. [PubMed: 6828866]

35. Verney C, Takahashi T, Bhide PG, Nowakowski RS, Caviness VS Jr. Independent controls for neocortical neuron production and histogenetic cell death. Dev Neurosci. 2000; 22:125-138. [PubMed: 10657705]

36. LaMantia AS, Rakic P. Axon overproduction and elimination in the anterior commissure of the developing rhesus monkey. J Comp Neurol. 1994; 340:328-336. [PubMed: 8188854]

37. LaMantia AS, Rakic P. Axon overproduction and elimination in the corpus callosum of the developing rhesus monkey. J Neurosci. 1990; 10:2156-2175. [PubMed: 2376772] 
38. Salami M, Itami C, Tsumoto T, Kimura F. Change of conduction velocity by regional myelination yields constant latency irrespective of distance between thalamus and cortex. Proc Natl Acad Sci U S A. 2003; 100:6174-6179. [PubMed: 12719546]

39. Barkovich AJ, Kjos BO, Jackson DE Jr, Norman D. Normal maturation of the neonatal and infant brain: MR imaging at 1.5 T. Radiology. 1988; 166(Pt 1):173-180. [PubMed: 3336675]

40. Ashtari M, Cervellione KL, Hasan KM, et al. White matter development during late adolescence in healthy males: a cross-sectional diffusion tensor imaging study. Neuroimage. 2007; 35:501-510. [PubMed: 17258911]

41. Grodd W. Normal and abnormal patterns of myelin development of the fetal and infantile human brain using magnetic resonance imaging. Curr Opin Neurol Neurosurg. 1993; 6:393-397. [PubMed: 8507909]

42. Thompson PM, Giedd JN, Woods RP, MacDonald D, Evans AC, Toga AW. Growth patterns in the developing brain detected by using continuum mechanical tensor maps. Nature. 2000; 404:190193. [PubMed: 10724172]

43. Lenglet C, Campbell JS, Descoteaux M, Haro G, Savadjiev P, Wassermann D, et al. Mathematical methods for diffusion MRI processing. Neuroimage. 2009; 45 Suppl:S111-S122. [PubMed: 19063977]

44. Miller MI, Qiu A. The emerging discipline of computational functional anatomy. Neuroimage. 2009; 45 Suppl:S16-S39. [PubMed: 19103297]

45. Sundgren PC, Dong Q, Gomez-Hassan D, Mukherji SK, Maly P, Welsh R. Diffusion tensor imaging of the brain: review of clinical applications. Neuroradiology. 2004; 46:339-350. [PubMed: 15103435]

46. Trivedi R, Husain N, Rathore RK, et al. Correlation of diffusion tensor imaging with histology in the developing human frontal cerebrum. Dev Neurosci. 2009; 31:487-496. [PubMed: 19622880]

47. Hermoye L, Saint-Martin C, Cosnard G, et al. Pediatric diffusion tensor imaging: normal database and observation of the white matter maturation in early childhood. Neuroimage. 2006; 29:493504. [PubMed: 16194615]

48. Dubois J, Dehaene-Lambertz G, Perrin M, et al. Asynchrony of the early maturation of white matter bundles in healthy infants: quantitative landmarks revealed noninvasively by diffusion tensor imaging. Hum Brain Mapp. 2008; 29:14-27. [PubMed: 17318834]

49. Qiu D, Tan LH, Zhou K, Khong PL. Diffusion tensor imaging of normal white matter maturation from late childhood to young adulthood: voxel-wise evaluation of mean diffusivity, fractional anisotropy, radial and axial diffusivities, and correlation with reading development. Neuroimage. 2008; 41:223-232. [PubMed: 18395471]

50. Sporns O, Zwi JD. The small world of the cerebral cortex. Neuroinformatics. 2004; 2:145-162. [PubMed: 15319512]

51. Bassett DS, Bullmore E. Small-world brain networks. Neuroscientist. 2006; 12:512-523. [PubMed: 17079517]

52. Fan Y, Shi F, Smith JK, Lin W, Gilmore JH, Shen D. Brain anatomical networks in early human brain development. Neuroimage. 2010 [Epub ahead of print].

53. Gong G, Rosa-Neto P, Carbonell F, Chen ZJ, He Y, Evans AC. Age- and gender-related differences in the cortical anatomical network. J Neurosci. 2009; 29:15684-15693. [PubMed: 20016083]

54. Kakeda S, Korogi Y. The efficacy of a voxel-based morphometry on the analysis of imaging in schizophrenia, temporal lobe epilepsy, and Alzheimer's disease/mild cognitive impairment: a review. Neuroradiology. 2010; 52:711-721. [PubMed: 20495793]

55. Feldman HM, Yeatman JD, Lee ES, Barde LH, Gaman-Bean S. Diffusion tensor imaging: a review for pediatric researchers and clinicians. J Dev Behav Pediatr. 2010; 31:346-356. [PubMed: 20453582]

56. Huang H. Delineating neural structures of developmental human brains with diffusion tensor imaging. Scientific World Journal. 2010; 10:135-144. [PubMed: 20098957]

57. Wahl M, Barkovich AJ, Mukherjee P. Diffusion imaging and tractography of congenital brain malformations. Pediatr Radiol. 2010; 40:59-67. [PubMed: 19937239] 
58. Ponten SC, Daffertshofer A, Hillebrand A, Stam CJ. The relationship between structural and functional connectivity: graph theoretical analysis of an EEG neural mass model. Neuroimage. 2010; 52:985-994. [PubMed: 19853665]

59. Hubel DH, Wiesel TN, LeVay S. Plasticity of ocular dominance columns in monkey striate cortex. Philos Trans R Soc Lond B Biol Sci. 1977; 278:377-409. [PubMed: 19791]

60. Uhlhaas PJ, Roux F, Rodriguez E, Rotarska-Jagiela A, Singer W. Neural synchrony and the development of cortical networks. Trends Cogn Sci. 2010; 14:72-80. [PubMed: 20080054]

61. Hebb, D. The Organization of Behavior: A Neuropsychological Theory. New York: Wiley; 1949.

62. Chu CJ, Jones TA. Experience-dependent structural plasticity in cortex heterotopic to focal sensorimotor cortical damage. Exp Neurol. 2000; 166:403-414. [PubMed: 11085905]

63. Komuro H, Rakic P. Modulation of neuronal migration by NMDA receptors. Science. 1993; 260:95-97. [PubMed: 8096653]

64. Rakic P, Komuro H. The role of receptor/channel activity in neuronal cell migration. J Neurobiol. 1995; 26:299-315. [PubMed: 7775964]

65. Katz LC, Shatz CJ. Synaptic activity and the construction of cortical circuits. Science. 1996; 274:1133-1138. [PubMed: 8895456]

66. Holmes GL, McCabe B. Brain development and generation of brain pathologies. Int Rev Neurobiol. 2001; 45:17-41. [PubMed: 11130898]

67. Boersma M, Smit DJ, de Bie HM, Van Baal GC, Boomsma DI, de Geus EJ, et al. Network analysis of resting state EEG in the developing young brain: structure comes with maturation. Hum Brain Mapp. 2010

68. Srinivasan R. Spatial structure of the human alpha rhythm: global correlation in adults and local correlation in children. Clin Neurophysiol. 1999; 110:1351-1362. [PubMed: 10454270]

69. Barry RJ, Clarke AR, McCarthy R, Selikowitz M, Johnstone SJ, Rushby JA. Age and gender effects in EEG coherence: I. Developmental trends in normal children. Clin Neurophysiol. 2004; 115:2252-2258. [PubMed: 15351366]

70. Marosi E, Harmony T, Sanchez L, et al. Maturation of the coherence of EEG activity in normal and learning-disabled children. Electroencephalogr Clin Neurophysiol. 1992; 83:350-357. [PubMed: 1281080]

71. Nunes ML, Da Costa JC, Moura-Ribeiro MV. Polysomnographic quantification of bioelectrical maturation in preterm and fullterm newborns at matched conceptional ages. Electroencephalogr Clin Neurophysiol. 1997; 102:186-191. [PubMed: 9129574]

72. Milh M, Kaminska A, Huon C, Lapillonne A, Ben-Ari Y, Khazipov R. Rapid cortical oscillations and early motor activity in premature human neonate. Cereb Cortex. 2007; 17:1582-1594. [PubMed: 16950867]

73. Uhlhaas PJ, Roux F, Singer W, Haenschel C, Sireteanu R, Rodriguez E. The development of neural synchrony reflects late maturation and restructuring of functional networks in humans. Proc Natl Acad Sci U S A. 2009; 106:9866-9871. [PubMed: 19478071]

74. Werkle-Bergner M, Shing YL, Muller V, Li SC, Lindenberger U. EEG gamma-band synchronization in visual coding from childhood to old age: evidence from evoked power and inter-trial phase locking. Clin Neurophysiol. 2009; 120:1291-1302. [PubMed: 19482545]

75. Buckner RL, Andrews-Hanna JR, Schacter DL. The brain's default network: anatomy, function, and relevance to disease. Ann N Y Acad Sci. 2008; 1124:1-38. [PubMed: 18400922]

76. Kennedy DP, Courchesne E. Functional abnormalities of the default network during self- and other-reflection in autism. Soc Cogn Affect Neurosci. 2008; 3:177-190. [PubMed: 19015108]

77. Damoiseaux JS, Rombouts SA, Barkhof F, et al. Consistent resting-state networks across healthy subjects. Proc Natl Acad Sci U S A. 2006; 103:13848-13853. [PubMed: 16945915]

78. Greicius MD, Krasnow B, Reiss AL, Menon V. Functional connectivity in the resting brain: a network analysis of the default mode hypothesis. Proc Natl Acad Sci U S A. 2003; 100:253-258. [PubMed: 12506194]

79. Cole DM, Smith SM, Beckmann CF. Advances and pitfalls in the analysis and interpretation of resting-state FMRI data. Front Syst Neurosci. 2010; 4:8. [PubMed: 20407579] 
80. Fransson P, Skiold B, Horsch S, et al. Resting-state networks in the infant brain. Proc Natl Acad Sci U S A. 2007; 104:15531-15536. [PubMed: 17878310]

81. Larson-Prior LJ, Zempel JM, Nolan TS, Prior FW, Snyder AZ, Raichle ME. Cortical network functional connectivity in the descent to sleep. Proc Natl Acad Sci U S A. 2009; 106:4489-4494. [PubMed: 19255447]

82. Smyser CD, Inder TE, Shimony JS, et al. Longitudinal analysis of neural network development in preterm infants. Cereb Cortex. 2010 [Epub ahead of print].

83. Fair DA, Cohen AL, Dosenbach NU, et al. The maturing architecture of the brain's default network. Proc Natl Acad Sci U S A. 2008; 105:4028-4032. [PubMed: 18322013]

84. Homae F, Watanabe H, Otobe T, et al. Development of global cortical networks in early infancy. J Neurosci. 2010; 30:4877-4882. [PubMed: 20371807]

85. Dosenbach NU, Fair DA, Miezin FM, et al. Distinct brain networks for adaptive and stable task control in humans. Proc Natl Acad Sci U S A. 2007; 104:11073-11078. [PubMed: 17576922]

86. Fair DA, Dosenbach NU, Church JA, et al. Development of distinct control networks through segregation and integration. Proc Natl Acad Sci U S A. 2007; 104:13507-13512. [PubMed: 17679691]

87. Supekar K, Musen M, Menon V. Development of large-scale functional brain networks in children. PLoS Biol. 2009; 7:e1000157. [PubMed: 19621066]

88. Thatcher RW, North DM, Biver CJ. Development of cortical connections as measured by EEG coherence and phase delays. Hum Brain Mapp. 2008; 29:1400-1415. [PubMed: 17957703]

89. Fair DA, Cohen AL, Power JD, et al. Functional brain networks develop from a "local to distributed" organization. PLoS Comput Biol. 2009; 5:e1000381. [PubMed: 19412534]

90. Gozzo Y, Vohr B, Lacadie C, Hampson M, Katz KH, Maller-Kesselman J, et al. Alterations in neural connectivity in preterm children at school age. Neuroimage. 2009; 48:458-463. [PubMed: 19560547]

91. Church JA, Fair DA, Dosenbach NU, et al. Control networks in paediatric Tourette syndrome show immature and anomalous patterns of functional connectivity. Brain. 2009; 132(Pt 1):225-238. [PubMed: 18952678]

92. Cao X, Cao Q, Long X, et al. Abnormal resting-state functional connectivity patterns of the putamen in medication-naive children with attention deficit hyperactivity disorder. Brain Res. 2009; 1303:195-206. [PubMed: 19699190]

93. Rubia K, Halari R, Cubillo A, Mohammad AM, Brammer M, Taylor E. Methylphenidate normalises activation and functional connectivity deficits in attention and motivation networks in medication-naive children with ADHD during a rewarded continuous performance task. Neuropharmacology. 2009; 57:640-652. [PubMed: 19715709]

94. Minshew NJ, Keller TA. The nature of brain dysfunction in autism: functional brain imaging studies. Curr Opin Neurol. 2010; 23:124-130. [PubMed: 20154614]

95. Alexander AL, Lee JE, Lazar M, et al. Diffusion tensor imaging of the corpus callosum in Autism. Neuroimage. 2007; 34:61-73. [PubMed: 17023185]

96. Lee JE, Bigler ED, Alexander AL, et al. Diffusion tensor imaging of white matter in the superior temporal gyrus and temporal stem in autism. Neurosci Lett. 2007; 424:127-132. [PubMed: 17714869]

97. Kana RK, Keller TA, Cherkassky VL, Minshew NJ, Just MA. Atypical frontal-posterior synchronization of Theory of Mind regions in autism during mental state attribution. Soc Neurosci. 2009; 4:135-152. [PubMed: 18633829]

98. Ebisch SJ, Gallese V, Willems RM, et al. Altered intrinsic functional connectivity of anterior and posterior insula regions in high-functioning participants with autism spectrum disorder. Hum Brain Mapp. 2010 [Epub ahead of print].

99. Assaf M, Jagannathan K, Calhoun VD, et al. Abnormal functional connectivity of default mode sub-networks in autism spectrum disorder patients. Neuroimage. 2010; 53:247-256. [PubMed: 20621638]

100. Monk CS, Peltier SJ, Wiggins JL, et al. Abnormalities of intrinsic functional connectivity in autism spectrum disorders. Neuroimage. 2009; 47:764-772. [PubMed: 19409498] 
101. Weng SJ, Wiggins JL, Peltier SJ, et al. Alterations of resting state functional connectivity in the default network in adolescents with autism spectrum disorders. Brain Res. 2010; 1313:202-214. [PubMed: 20004180]

102. Siniatchkin M, Groening K, Moehring J, et al. Neuronal networks in children with continuous spikes and waves during slow sleep. Brain. 2010; 133:2798-2813. [PubMed: 20688812] 


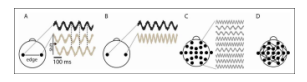

Figure 1.

Example illustration of constructing functional networks from voltage signals. (A) In this simulated example, electroencephalogram (EEG) data are recorded from 2 electrodes on the scalp surface. The EEG data (black and upper gray curve) are simple sinusoids with added noise, and the lower gray trace is the middle trace shifted to the right by $\sim 25 \mathrm{~ms}$ - this time advance of the EEG signal causes it to align with the top (black) trace, indicated by the vertical dashed lines. Because the 2 signals align, the cross correlation is strong enough, and we connect the 2 electrodes with an edge (black line) to form a simple 2-node network. (B) In this contrasting example, the EEG data never match, no matter the choice of time shift, and the 2-node network lacks an edge. (C) Simulated multivariate data recorded from many EEG electrodes, results in (D) a much more complicated network. 


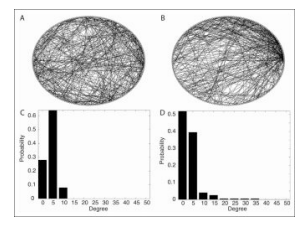

Figure 2.

Two larger networks consisting of 200 nodes. (A,B) We arrange the nodes (gray) in the network as a ring, although this does not suggest a literal spatial sensor location in the "real" recording space. Each node represents an individual electrode or sensor, and each edge (black line) indicates sufficiently strong coupling between activity recorded simultaneously at 2 nodes. For such large networks, the network structure becomes much more difficult to characterize through visual inspection. (C,D) The degree distributions for each network. For the network in (A) most of the nodes have a degree near 5. For the network in (B), most of the nodes have a degree less than 5 , but some nodes have a high degree (up to 35 edges). 


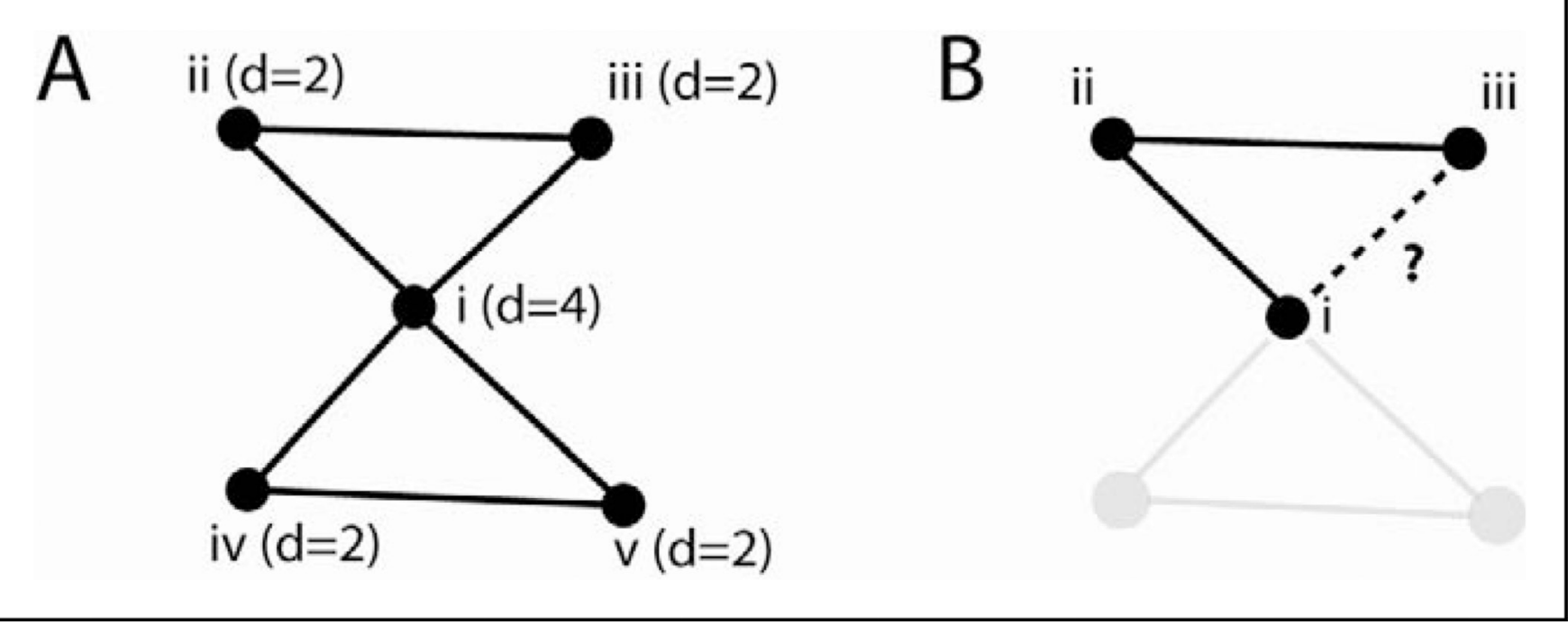

Figure 3.

An example 5-node network to illustrate 3 simple measures of network structure. (A) We label the 5-nodes (circles) with roman numerals and connect the nodes with edges (lines). The number in parentheses next to each node indicates its degree (d). (B) To determine the clustering coefficient of node ii, we first determine its nearest neighbors (the other 2 nodes in the network are grayed out). We then determine if an edge exists between these neighbors (the dotted line). Because this edge exists in (A), the 3 nodes form a triangle or cluster. 


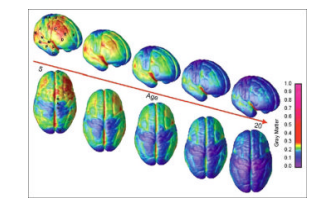

Figure 4.

Dynamic sequence of gray matter maturation over the cortical surface from age 5 to 20 years, demonstrating maturation of primary motor and sensory regions before association cortices and prefrontal cortices. (Reprinted with permission from Gogtay N, Giedd JN, Lusk L, et al. Dynamic mapping of human cortical development during childhood through early adulthood. Proc Natl Acad Sci U S A. 2004;101:8174-8179. Copyright 2004 National Academy of Sciences.) 


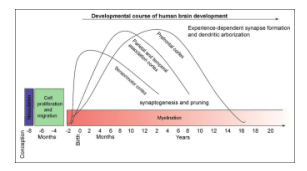

Figure 5 .

Observed timeline of some of the progressive and regressive structural changes present over the course of prenatal through adolescent brain development. This figure can be taken to represent the general hierarchical elaboration of forebrain neural systems where the events in primary representations anticipate those in successively more integrative regions. ${ }^{33}$ (Reprinted with permission from Casey BJ, Tottenham N, Liston C, Durston S. Imaging the developing brain: what have we learned about cognitive development? Trends Cogn Sci. 2005;9:104-110. Copyright 2005 Elsevier Ltd.) 


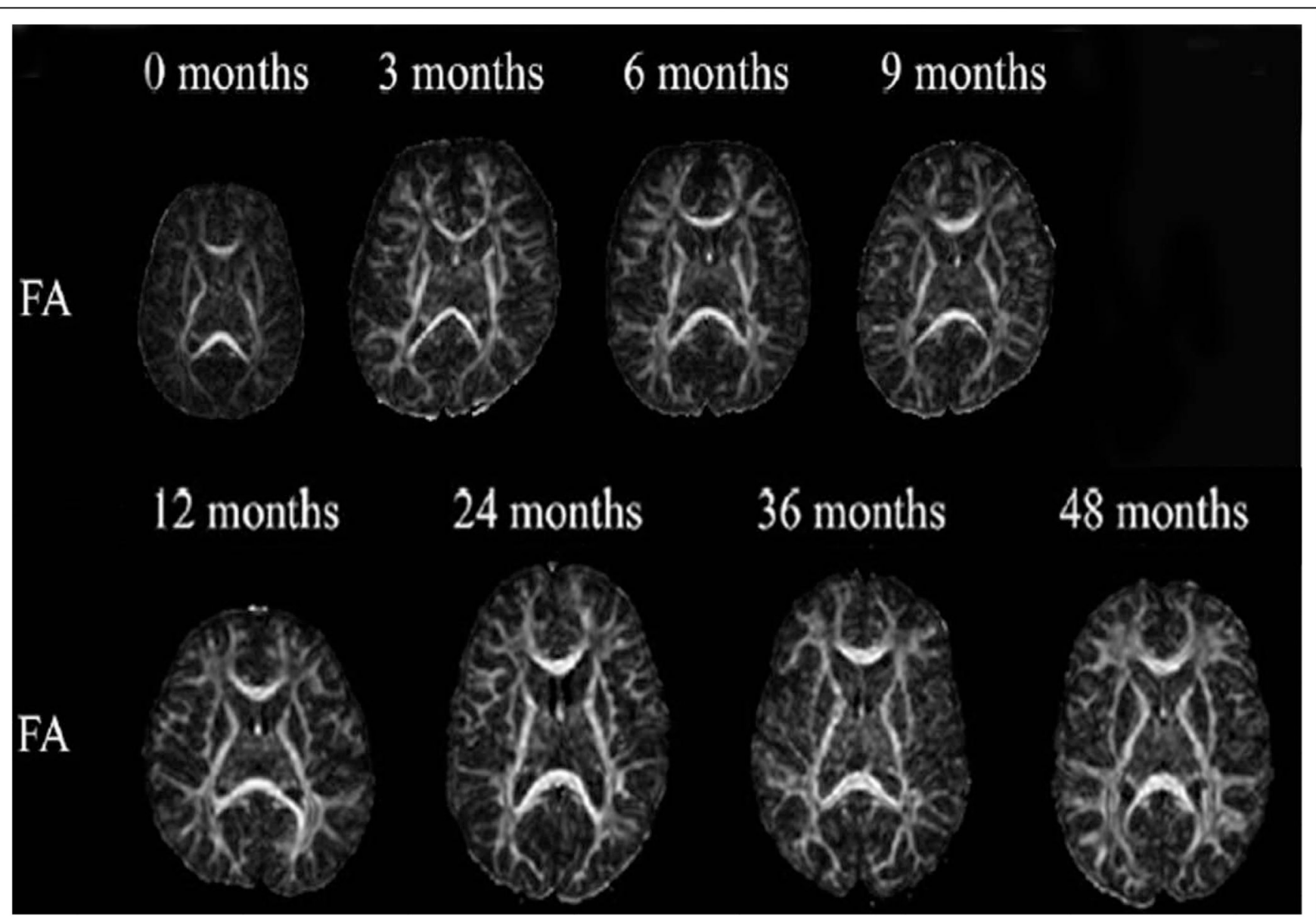

Figure 6.

Representative axial fractional anisotropy maps at $0,3,6,9,12,24,36$, and 48 months. (Reprinted with permission from Hermoye L, Saint-Martin C, Cosnard G, et al. Pediatric diffusion tensor imaging: normal database and observation of the white matter maturation in early childhood. Neuroimage. 2006;29:493-504. Copyright 2006 Elsevier, Ltd.) 


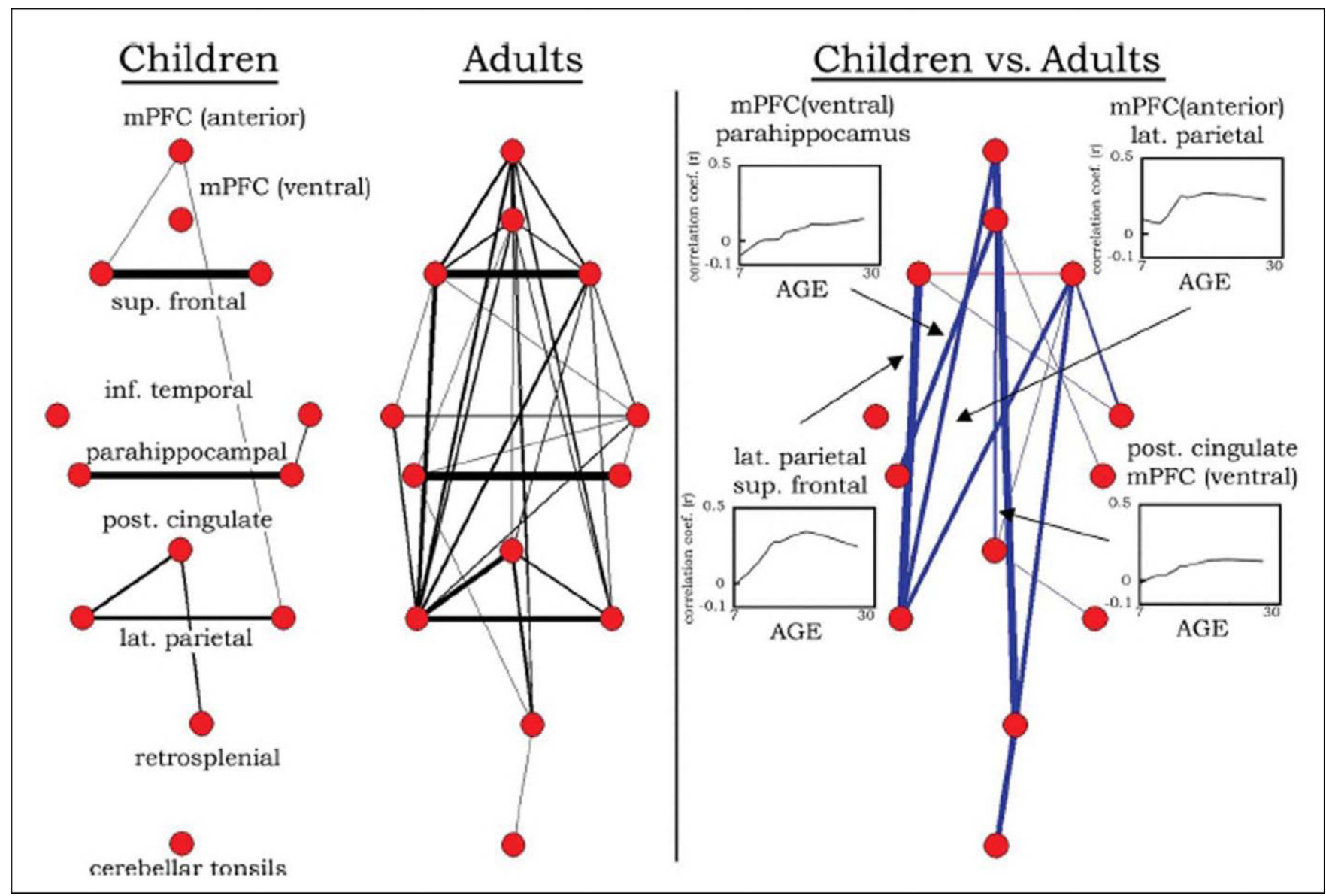

Figure 7.

Graph visualization of the correlation between default network regions in children (aged 7-9 years) and in adults (aged 21-31 years) represented in pseudoanatomical organization. Statistically significant differences in functional connectivity between children and adults are highlighted on the right. Connections between interhemispheric homotopic regions are relatively prominent in both children and adults, however the default network is fragmented with sparse connections in children compared with adults. (Adapted with permission from Fair DA, Cohen AL, Dosenbach NU, et al. The maturing architecture of the brain's default network. Proc Natl Acad Sci U S A. 2008;105: 4028-4032. Copyright 2008 National Academy of Sciences.) 